\title{
REMARKS ON THE REGULARITY OF QUASISLITS
}

\author{
Lukas Schoug, Atul Shekhar and Fredrik Viklund \\ University of Cambridge, Faculty of Mathematics \\ Wilberforce Road, Cambridge CB3 0WA, United Kingdom; lschoug@maths.cam.ac.uk \\ Université Lyon 1, Département Mathématiques \\ 69622 Villeurbanne Cedex, France; atulshekhar83@gmail.com \\ KTH Royal Institute of Technology, Department of Mathematics \\ 10044 Stockholm, Sweden; fredrik.viklund@math.kth.se
}

\begin{abstract}
A quasislit is the image of a vertical line segment $[0, i y], y>0$, under a quasiconformal homeomorphism of the upper half-plane fixing $\infty$. Quasislits correspond precisely to curves generated by the Loewner equation with a driving function in the Lip- $\frac{1}{2}$ class. It is known that a quasislit is contained in a cone depending only on its Loewner driving function Lip- $\frac{1}{2}$ seminorm, $\sigma$. In this note we use the Loewner equation to give quantitative estimates on the opening angle of this cone in the maximal range $\sigma<4$. The estimate is shown to be sharp for small $\sigma$. As consequences, we derive explicit Hölder exponents for $\sigma<4$ as well as estimates on winding rates. We also relate quantitatively the Lip- $\frac{1}{2}$ seminorm with the quasiconformal dilatation and discuss the optimal regularity of quasislits achievable through reparametrization.
\end{abstract}

\section{Introduction}

A quasicircle is the image of the unit circle under a quasiconformal homeomorphism of the complex plane. Recall that $f \in W_{\text {loc }}^{1,2}$ is a $k$-quasiconformal map if it is a homeomorphic solution to the Beltrami equation, $\bar{\partial} f=\mu(z) \partial f$, where $\mu$ is measurable, complex valued, and such that $k:=\|\mu\|_{\infty}<1$ with corresponding maximal dilatation $(1+k) /(1-k)$. Many equivalent characterizations of quasicircles exist, both analytic and geometric, see e.g. [3]. For a particular characterization it is quite natural to ask how the geometry of the quasicircle depends quantitatively on the given data and how the data for different characterizations are related. For example, a $k$-quasicircle is the image of a $k$-quasiconformal map and a well-known theorem of Smirnov (motivated by a conjecture of Astala) states that the dimension of such a quasicircle is at most $1+k^{2}$ for $k$ small. ${ }^{1}$

In this note we will quantify some simple geometric features of quasicircles seen from the point of view of the Loewner equation. In this case it is more convenient to consider quasislits, that is, the image of a line segment $\left\{i y: 0 \leq y \leq y_{0}\right\} \quad\left(y_{0} \in\right.$ $(0, \infty])$ under a quasiconformal homeomorphism $\mathbf{H} \rightarrow \mathbf{H}$, fixing $\infty$. Every quasislit has a Loewner driving function in the Lip- $\frac{1}{2}$ class (see below) and conversely, every continuous function is the Loewner driving function for a quasislit if it is in the Lip- $\frac{1}{2}$ class with small seminorm. We will primarily be interested in understanding how properties of the curve depend quantitatively on this seminorm.

1.1. Curves, the Loewner equation, quasiarcs, and Lip- $\frac{1}{2}$. A curve is an equivalence class of continuous functions $[0,1] \rightarrow \mathbf{C}$, where two representatives are

https://doi.org/10.5186/aasfm.2021.4623

2020 Mathematics Subject Classification: Primary 30C99.

Key words: Loewner differential equation, quasicircles, quasislits.

${ }^{1}$ Recent work of Ivrii shows however that this bound is not sharp, see Section 6 . 
in the same equivalence class if and only if each one can be obtained from the other by an increasing reparametrization. That is, $\gamma_{1}$ and $\gamma_{2}$ describe the same curve if and only if there exist increasing homeomorphisms $\alpha_{1}, \alpha_{2}:[0,1] \rightarrow[0,1]$ such that $\gamma_{1} \circ \alpha_{1}(t)=\gamma_{2} \circ \alpha_{2}(t), t \in[0,1]$. We will almost always consider curves with a particular parametrization chosen and refer to this as a curve as well.

Let $\lambda_{t}=\lambda(t), t \in[0,1]$, be a continuous real-valued function with $\lambda_{0}=0$, and consider for $z \in \mathbf{H}=\{z: \operatorname{Im} z>0\}$ the solution $\left(f_{t}(z)\right)$ to the Loewner PDE

$$
\partial_{t} f_{t}(z)=-f_{t}^{\prime}(z) \frac{2}{z-\lambda_{t}}, \quad f_{0}(z)=z .
$$

The family $\left(f_{t}\right)$ is called a Loewner chain and for each $t, f_{t}: \mathbf{H} \rightarrow H_{t} \subset \mathbf{H}$ is a conformal map normalized 'hydrodynamically' at $\infty$ by $f_{t}(z)=z-2 t / z+O\left(1 /|z|^{2}\right)$. If $\lambda_{t}$ is sufficiently well-behaved, the limit

$$
\gamma(t)=\lim _{y \downarrow 0} f_{t}\left(\lambda_{t}+i y\right)
$$

exists for every $t \in[0,1]$, defines a continuous function $t \mapsto \gamma(t)$, and the simply connected domain $H_{t}$ is the unbounded connected component of $\mathbf{H} \backslash \gamma[0, t]$. In this case, we say that the Loewner chain is generated by the curve $\gamma$. Viewed differently, the driving term $\lambda$ generates the (chordal) Loewner curve $\gamma=\gamma^{\lambda}$ which comes equipped with a particular parametrization from the Loewner equation via (1.2) called the (half-plane) capacity parametrization. This process can be reversed, and starting from, e.g., a simple curve in $\overline{\mathbf{H}}$ meeting $\mathbf{R}$ non-tangentially at its starting point, otherwise staying in $\mathbf{H}$, one can parametrize by capacity and recover its driving term from the hydrodynamically normalized uniformizing conformal maps $f_{t}^{-1}: \mathbf{H} \backslash$ $\gamma[0, t] \rightarrow \mathbf{H}$.

Remark. Applying $z \mapsto z^{2}$, and 'completing' the resulting curve in union with $\mathbf{R}_{+}$by a hyperbolic geodesic from the endpoint to $\infty$, we can naturally think of a Loewner curve as part of a loop in $\hat{\mathbf{C}}$ through 0 and $\infty$ containing $\mathbf{R}_{+}$as a sub-arc. In fact, any simple loop in $\hat{\mathbf{C}}$ (that does not necessarily contain $\mathbf{R}_{+}$as a sub-arc) can be described by a two-sided Loewner equation, driven by a function defined on $\mathbf{R}$, see Section 6 of [16].

Here we will be interested in curves corresponding to driving terms in the Lip- $\frac{1}{2}$ class, that is, functions satisfying

$$
\|\lambda\|_{\frac{1}{2}}:=\sup _{s \neq t} \frac{\left|\lambda_{t}-\lambda_{s}\right|}{|t-s|^{\frac{1}{2}}}<\infty .
$$

For $\sigma>0$, write

$$
\Lambda_{\sigma}=\left\{\lambda:[0,1] \rightarrow \mathbf{R} \mid\|\lambda\|_{\frac{1}{2}} \leq \sigma, \lambda_{0}=0\right\} .
$$

The fundamental observation is due to Marshall and Rohde [11]: there exists $C$ so that if $\lambda \in \Lambda_{\sigma}$ with $\sigma<C$ then $\gamma^{\lambda}$ is a quasislit, in particular a simple curve. Lind showed that one can take $C=4$ and that this is sharp in the sense that for each $\sigma \geq 4$, there exists a function $\lambda \in \Lambda_{\sigma}$ that does not even generate a curve [8]. Conversely, if $\gamma\left[0, t_{0}\right]$ is a quasislit generated by $\lambda$, then $\lambda_{t}, t \in\left[0, t_{0}\right]$, is in Lip- $\frac{1}{2}$. See $[9,10,13]$ for more. For later reference we note that an equivalent description of a quasislit is as a quasiarc (i.e., the image of a line segment under a quasiconformal homeomorphism of $\mathbf{C}$ ) in $\overline{\mathbf{H}}$ that meets $\mathbf{R}$ non-tangentially at its starting point and otherwise stays in $\mathbf{H}$. 
We may now define

$$
\Gamma_{\sigma}=\left\{\gamma^{\lambda} \mid \lambda \in \Lambda_{\sigma}\right\}, \quad \sigma<4
$$

and in what follows, we will consider quasislits $\gamma \in \Gamma_{\sigma}$.

1.2. Results. For $\sigma<4$ and $t \geq 0$, define

$$
\mathcal{C}_{t, \sigma}=\sup _{\gamma \in \Gamma_{\sigma}} \frac{|\operatorname{Re}(\gamma(t))|}{\operatorname{Im}(\gamma(t))}
$$

This quantity measures the smallest opening angle required so that a cone at 0 contains any curve in $\Gamma_{\sigma}$. Our goal is to estimate $\mathcal{C}_{t, \sigma}$ and then derive regularity information from this bound. In order to state the result, fix $\sigma<4$ and consider the following equation

$$
e^{x}=\frac{\sqrt{16-\sigma^{2}}}{\sqrt{16 x^{2}-\sigma^{2}(x+1)^{2}}} .
$$

The right-hand side of (1.3) is defined for $x>\sigma /(4-\sigma)$. It is a continuous function which is strictly decreasing from $\infty$ to 0 as $x$ ranges from $\sigma /(4-\sigma)$ to $\infty$. Therefore, the equation has a unique solution, which we will denote by $p(\sigma)$. Next, define

$$
L_{\sigma}=\frac{\sigma}{\sqrt{16-\sigma^{2}}}(1+p(\sigma)) e^{p(\sigma)} .
$$

We have the following.

Theorem 1.1. The following bounds hold uniformly in $t>0$ :

(i) If $0<\sigma<4$, then

(ii) If $0<\sigma<\frac{8}{\pi}$, then

$$
\mathcal{C}_{t, \sigma} \leq L_{\sigma}
$$

$$
\mathcal{C}_{t, \sigma} \leq \frac{\pi \sigma}{\sqrt{64-\pi^{2} \sigma^{2}}}
$$

We prove (ii) in Section 3 and (i) in Section 4.

The function $L_{\sigma}$ has the following expansion as $\sigma \rightarrow 0$ :

$$
L_{\sigma}=\frac{1}{4}\left(1+\frac{1}{\mathcal{W}(1)}\right) \sigma+O\left(\sigma^{3}\right)
$$

where $\mathcal{W}(\cdot)$ is the Lambert $W$ function, so that $L_{\sigma} \sim 0.69 \sigma$ as $\sigma \rightarrow 0$. Comparing with (ii) we see that the estimate in (i) is not sharp, but does provide the first nontrivial and explicit bound that holds for all $\sigma<4$. On the other hand, by choosing the driving function $\lambda_{t}=\sigma \sqrt{t} \in \Lambda_{\sigma}$ we see that for all $t>0$

$$
\mathcal{C}_{t, \sigma} \geq \tan \left(\frac{\pi}{2} \frac{\sigma}{\sqrt{16+\sigma^{2}}}\right)
$$

see Example 4.12 in [7]. This shows that the estimate (ii) of Theorem 1.1 is sharp for small $\sigma$, and we have

$$
\mathcal{C}_{t, \sigma}=\frac{\pi}{8} \sigma+O\left(\sigma^{3}\right)
$$

Let us discuss some consequences of Theorem 1.1. Suppose $\gamma \in \Gamma_{\sigma}$ with $\sigma<$ 4. Then by [13], Remark 4.2, there exists $\beta_{\sigma} \in\left(0, \frac{1}{2}\right]$, depending only on $\sigma$, such that $\gamma(t), t \in[0,1]$, parametrized by half-plane capacity is Hölder continuous with exponent $\beta_{\sigma}$. Time 0 is special for Loewner curves in $\mathbf{H}$ parametrized by capacity: under weak assumptions, such curves are always Hölder- $\frac{1}{2}$ at $t=0$, see, e.g., [6]. To achieve better regularity one can restrict attention to strictly positive times (or 
consider driving terms that are constant during a small time interval starting with $0)$. Let us fix $\varepsilon>0$ and given a Loewner curve $\gamma$ we consider its optimal Hölder exponent for the capacity parametrization

$$
\alpha(\gamma)=\sup \{\alpha: \gamma \text { parametrized by capacity is Hölder- } \alpha \text { on }[\varepsilon, 1]\} .
$$

(If $\gamma$ is not Hölder continuous in the capacity parmetrization, by convention $\alpha(\gamma)=$ 0.) Next, define the corresponding universal exponent for the class $\Gamma_{\sigma}$ by

$$
\alpha\left(\Gamma_{\sigma}\right)=\inf \left\{\alpha(\gamma): \gamma \in \Gamma_{\sigma}\right\}
$$

It is not hard to show that if $\sigma<2$, then one has $\alpha\left(\Gamma_{\sigma}\right) \geq 1-\sigma^{2} / 4$, see, e.g., [13], but this bound is not sharp and in the range $\sigma \in[2,4)$ no estimate except $\alpha\left(\Gamma_{\sigma}\right)>0$ was as far as we are aware known prior to this paper. This is in contrast with the much rougher setting of SLE where the sharp Hölder exponents for the capacity parametrization are known [6]. In this case, the randomness helps in the analysis, see below for further discussion.

Our first corollary is a quantitative estimate on $\alpha\left(\Gamma_{\sigma}\right)$ for $\sigma \in[0,4)$.

Corollary 1.2. If $\sigma<4$, then

$$
\alpha\left(\Gamma_{\sigma}\right) \geq \frac{1}{1+L_{\sigma}^{2}}
$$

where $L_{\sigma}$ is given by (1.4).

Our second corollary improves on the regularity estimate (1.6) (and the easy one $\left.\alpha\left(\Gamma_{\sigma}\right) \geq 1-\sigma^{2} / 4\right)$, in the smaller range $\sigma \in(0,8 / \pi)$.

Corollary 1.3. If $\sigma<\frac{8}{\pi}$, then

$$
\alpha\left(\Gamma_{\sigma}\right) \geq 1-\frac{\pi^{2} \sigma^{2}}{64} .
$$

The proofs of these corollaries are the same, and given in Section 5.1.

Even though the cone estimate is sharp for small $\sigma$, we do not expect the exponents of Corollary 1.3 to be sharp, see Section 5.1. Here we simply remark that choosing $\lambda_{t}=\sigma \sqrt{1-t}$ produces a curve with a spiral at $t=1$ and a Hölder exponent of $1-\sigma^{2} / 16$. Indeed, this can be seen from direct computation using the representation given in Proposition 3.3 of [9], see, e.g., [12]. So $\alpha\left(\Gamma_{\sigma}\right) \leq 1-\sigma^{2} / 16$ and Rohde and Viklund have conjectured that this is in fact sharp for $\sigma \in[0,4)$, but we currently have no proof of a matching lower bound.

Remark. Let $D\left(\Gamma_{\sigma}\right)$ be the maximal Hausdorff dimension of $\gamma \in \Gamma_{\sigma}$. Corollary 1.3 immediately implies

$$
D\left(\Gamma_{\sigma}\right) \leq 1+\pi^{2} \sigma^{2} / 64+O\left(\sigma^{4}\right)
$$

as $\sigma \rightarrow 0$.

Theorem 1.1 also gives non-trivial bounds on winding rates for the curve near the tip, when $\sigma$ is small, see Section 5.2.

In Section 6 we make a few simple observations about the related problem of estimating the optimal Hölder regularity for quasislits achievable through reparametrization and the relation between the quasiconformal dilatiation parameter $k$ and the semi-norm $\sigma$. 
Acknowledgements. LS acknowledges support from the Knut and Alice Wallenberg foundation and a previous version of this paper was a part of LS's KTH Royal Institute of Technology doctoral thesis. AS acknowledges the support from the Gustafsson foundation and European Research Council (ERC) grant LiKo 676999. FV acknowledges support from the Knut and Alice Wallenberg foundation, the Swedish Research Council, and the Gustafsson foundation.

Thanks go to the referee for several useful comments. We thank Yilin Wang for discussions and comments on an earlier version of our paper. FV would like to thank Steffen Rohde for many inspiring discussions about the topics covered in this paper.

\section{Preliminaries}

In this section we recall some basic properties of the Loewner differential equation and related objects which we will need in our analysis. Recall that $g_{t}=f_{t}^{-1}$ satisfies a family of ODEs,

$$
\partial_{t} g_{t}(z)=\frac{2}{g_{t}(z)-\lambda_{t}}, \quad g_{0}(z)=z .
$$

The family $\left(g_{t}\right)$ is also called a Loewner chain. We will frequently write expressions of the form $d Z_{t}=a(t) d F(t)$ for functions $a, F$, which are to be interpreted as

$$
\int_{t_{0}}^{t} d Z_{s}=\int_{t_{0}}^{t} a(s) d F(s) .
$$

Throughout, we let $\sigma<4$ and assume that $\gamma$ is generated by $\lambda \in \Lambda_{\sigma}$ and that $\left(g_{t}\right)_{t \geq 0}$ is the corresponding Loewner chain. To analyze $f_{t}$, which satisfies a PDE, it is convenient to use the reverse flow. For fixed $t>0$ and $s \in[0, t]$, let $\beta_{s}^{t}=\lambda_{t}-\lambda_{t-s}$. If $t$ is understood from the context, we instead write $\beta_{s}$. Then the following holds.

Lemma 2.1. Fix $t>0$ and let $\hat{h}_{s}$ satisfy the equation

$$
d \hat{h}_{s}(z)=d \beta_{s}^{t}-\frac{2}{\hat{h}_{s}(z)} d s, \quad \hat{h}_{0}(z)=z,
$$

for $s \in[0, t]$ and $z \in \mathbf{H}$. Then

$$
\hat{h}_{t}(z)=f_{t}\left(\lambda_{t}+z\right) \text {. }
$$

Proof. Use (2.1) to see that the family of maps $\hat{h}_{s}$, defined by $\hat{h}_{s}(z):=g_{t-s}\left(f_{t}(z+\right.$ $\left.\left.\lambda_{t}\right)\right)-\lambda_{t-s}$ for $0 \leq s \leq t$, satisfies $(2.2)$ and $\hat{h}_{t}(z)=f_{t}\left(\lambda_{t}+z\right)$.

Remark. Note that $\hat{h}_{s}(z)$ depends on $t$ : if we let $\tilde{h}_{s}$ solve $(2.2)$ with driving function $\lambda$ but for $t_{1} \neq t$ in the same way, then it does not hold in general that $\hat{h}_{s}(z)=\tilde{h}_{s}(z)$ for $s<\min \left(t, t_{1}\right)$.

If we write $\hat{h}_{s}(z)=X_{s}(z)+i Y_{s}(z)$ for $z=x+i y \in \mathbf{H}$, then (2.2) is equivalent to the equations

$$
\begin{aligned}
& d X_{s}=d \beta_{s}-\frac{2 X_{s}}{X_{s}^{2}+Y_{s}^{2}} d s, \quad X_{0}=x \\
& d Y_{s}=\frac{2 Y_{s}}{X_{s}^{2}+Y_{s}^{2}} d s, \quad Y_{0}=y
\end{aligned}
$$

Next, we recall the following from [13]. 
Lemma 2.2. [13, Theorem 3.1 and Lemma 2.1] Suppose $\sigma<4$. There exists a constant $c_{\sigma}>0$, depending only on $\sigma$, such that for all $0<y \leq 1$ and $s \geq 0$,

$$
\sqrt{y^{2}+c_{\sigma} s} \leq Y_{s}(i y) \leq \sqrt{y^{2}+4 s}
$$

Moreover,

$$
\left|X_{s}(i y)\right| \leq \sup _{0 \leq r \leq s}\left|\beta_{s}-\beta_{r}\right| .
$$

In order to estimate $|\operatorname{Re}(\gamma(t))| / \operatorname{Im}(\gamma(t))$ we will work with the process

$$
W_{s}(z)=\frac{X_{s}(z)}{Y_{s}(z)}
$$

and note that by $(1.2)$

$$
\frac{\operatorname{Re}(\gamma(t))}{\operatorname{Im}(\gamma(t))}=\lim _{y \rightarrow 0} W_{t}(i y)
$$

Moreover, we can rewrite (2.3) as

$$
d X_{s}=d \beta_{s}-\frac{2 W_{s}^{2}}{W_{s}^{2}+1} \frac{1}{X_{s}} d s, \quad X_{0}=x .
$$

When estimating the Hölder exponents and the winding rates for the curves, we need to estimate $\left|f_{t}^{\prime}\left(\lambda_{t}+i y\right)\right|$ and $\arg f_{t}^{\prime}\left(\lambda_{t}+i y\right)$, where differentiation is with respect to the spatial variable. Since $f_{t}^{\prime}\left(\lambda_{t}+z\right)=\hat{h}_{t}^{\prime}(z)$ the following formulas for $\hat{h}_{s}^{\prime}(z)$ will be useful. For $s \in[0, t]$,

$$
\begin{aligned}
\left|\hat{h}_{s}^{\prime}(z)\right| & =\exp \left\{\int_{0}^{s} \frac{2\left(X_{r}^{2}-Y_{r}^{2}\right)}{\left(X_{r}^{2}+Y_{r}^{2}\right)^{2}} d r\right\}=\exp \left\{\int_{0}^{s} \frac{X_{r}^{2}-Y_{r}^{2}}{X_{r}^{2}+Y_{r}^{2}} d \log Y_{r}\right\} \\
& =\exp \left\{\int_{0}^{s} \frac{W_{r}^{2}-1}{W_{r}^{2}+1} d \log Y_{r}\right\}, \\
\arg \hat{h}_{s}^{\prime}(z) & =-4 \int_{0}^{s} \frac{X_{r} Y_{r}}{\left(X_{r}^{2}+Y_{r}^{2}\right)^{2}} d r=-2 \int_{0}^{s} \frac{X_{r} Y_{r}}{X_{r}^{2}+Y_{r}^{2}} d \log Y_{r} \\
& =-2 \int_{0}^{s} \frac{W_{r}}{W_{r}^{2}+1} d \log Y_{r} .
\end{aligned}
$$

2.1. Time reparametrization. By (2.4) and (2.5), it follows that $Y_{s}:[0, \infty) \rightarrow$ $[y, \infty)$ is a strictly increasing, continuous function and hence that the function $\left(Y_{s}^{2}-\right.$ $\left.y^{2}\right):[0, \infty) \rightarrow[0, \infty)$ is a bijection. We denote by $\theta_{s}$ its inverse function, that is, $Y_{\theta_{s}}^{2}-y^{2}=s$ and denote the reparametrized functions by $\tilde{X}_{s}=X_{\theta_{s}}, \tilde{Y}_{s}=Y_{\theta_{s}}=$ $\sqrt{y^{2}+s}$ and $\tilde{W}_{s}=\tilde{X}_{s} / \sqrt{y^{2}+s}$. Then we have

$$
\begin{aligned}
& d \tilde{X}_{s}=d \beta_{\theta_{s}}-\frac{\tilde{X}_{s}}{2\left(y^{2}+s\right)} d s, \quad \tilde{X}_{0}=x, \\
& d \theta_{s}=\frac{1}{4}\left(\frac{\tilde{X}_{s}^{2}}{y^{2}+s}+1\right) d s, \quad \theta_{0}=0 .
\end{aligned}
$$

The advantage of this reparametrization is that it only leaves us with one unknown: $\tilde{X}$. This makes it easier to compare two solutions with different driving functions and will play a crucial role in the proof of Theorem 1.1. 


\section{A bound in the range $0 \leq \sigma<\frac{8}{\pi}$}

This section is devoted to the proof of part (ii) of Theorem 1.1, and we will prove the following result, which will imply it.

Proposition 3.1. Let $\lambda \in \Lambda_{\sigma}$ with $\sigma<\frac{8}{\pi}$, fix some $t>0$ and define $\hat{h}_{s}(i y)=$ $X_{s}(i y)+i Y_{s}(i y)$ as the solution to (2.2). Then, for all $s \in[0, t]$ and $y>0$, we have

$$
\left|W_{s}(i y)\right|=\left|\frac{X_{s}(i y)}{Y_{s}(i y)}\right| \leq \frac{\pi \sigma}{\sqrt{64-\pi^{2} \sigma^{2}}} .
$$

Proof. Using the product formula together with (2.3) and (2.4), we see that

$$
d\left(X_{s} Y_{s}\right)=X_{s} d Y_{s}+Y_{s} d X_{s}=Y_{s} d \beta_{s}
$$

The validity of (3.2), interpreted as Riemann-Stieltjes integrals, follows since the continuity and monotonicity of $Y_{s}$ implies that it is of bounded variation. By partial integration, we have

$$
X_{s} Y_{s}=\int_{0}^{s} Y_{r} d \beta_{r}=y \beta_{s}+\int_{0}^{s}\left(\beta_{s}-\beta_{r}\right) d Y_{r}
$$

since $X_{0}=0$. Employing the time reparametrization of Section 2.1, using $d \tilde{Y}_{r}=$ $d r /\left(2 \sqrt{y^{2}+r}\right),(3.3)$ becomes

$$
\tilde{X}_{s} \sqrt{y^{2}+s}=y \beta_{\theta_{s}}+\int_{0}^{s}\left(\beta_{\theta_{s}}-\beta_{\theta_{r}}\right) \frac{d r}{2 \sqrt{y^{2}+r}} .
$$

In order to prove the result, we shall bound $\tilde{W}_{s}=\tilde{X}_{s} / \tilde{Y}_{s}=\tilde{X}_{s} / \sqrt{y^{2}+s}$. We have by $(3.4)$

$$
\tilde{W}_{s}=\frac{y \beta_{\theta_{s}}}{y^{2}+s}+\frac{1}{y^{2}+s} \int_{0}^{s}\left(\beta_{\theta_{s}}-\beta_{\theta_{r}}\right) \frac{d r}{2 \sqrt{y^{2}+r}} .
$$

We write $R_{s}=\sup _{r \leq s}\left|\tilde{W}_{r}\right|$ and note that by $(2.12)$ and $\|\beta\|_{\frac{1}{2}} \leq \sigma$,

$$
\left|\beta_{\theta_{s}}\right| \leq \sigma\left|\theta_{s}\right|^{\frac{1}{2}} \leq \frac{\sigma}{2} \sqrt{\int_{0}^{s}\left(\tilde{W}_{u}^{2}+1\right) d u} \leq \frac{\sigma \sqrt{s}}{2} \sqrt{R_{s}^{2}+1}
$$

and

$$
\begin{aligned}
\left|\int_{0}^{s}\left(\beta_{\theta_{s}}-\beta_{\theta_{r}}\right) \frac{d r}{2 \sqrt{y^{2}+r}}\right| & \leq \int_{0}^{s} \sigma \sqrt{\theta_{s}-\theta_{r}} \frac{d r}{2 \sqrt{y^{2}+r}} \\
& \leq \frac{\sigma}{2} \int_{0}^{s} \sqrt{\int_{r}^{s}\left(\tilde{W}_{u}^{2}+1\right) d u} \frac{d r}{2 \sqrt{y^{2}+r}} \\
& \leq \frac{\sigma}{2} \sqrt{R_{s}^{2}+1} \int_{0}^{s} \sqrt{s-r} \frac{d r}{2 \sqrt{y^{2}+r}}
\end{aligned}
$$


Next, we observe that

$$
\begin{aligned}
\int_{0}^{s} \sqrt{s-r} \frac{d r}{2 \sqrt{y^{2}+r}} & =\left(y^{2}+s\right) \int_{\frac{y}{\sqrt{y^{2}+s}}}^{1} \sqrt{1-u^{2}} d u \\
& =\left(y^{2}+s\right)\left(\frac{\pi}{4}-\int_{0}^{\frac{y}{\sqrt{y^{2}+s}}} \sqrt{1-u^{2}} d u\right) \\
& =\left(y^{2}+s\right)\left(\frac{\pi}{4}-I\left(\frac{y}{\sqrt{y^{2}+s}}\right)\right),
\end{aligned}
$$

where $I(x)=\frac{1}{2} \arcsin (x)+\frac{1}{2} x \sqrt{1-x^{2}}$. Putting together equations (3.5)-(3.8), we get

$$
\begin{aligned}
\left|\tilde{W}_{s}\right| & \leq \frac{y\left|\beta_{\theta_{s}}\right|}{y^{2}+s}+\frac{1}{y^{2}+s}\left|\int_{0}^{s}\left(\beta_{\theta_{s}}-\beta_{\theta_{r}}\right) \frac{d r}{2 \sqrt{y^{2}+r}}\right| \\
& \leq \frac{\sigma \sqrt{R_{s}^{2}+1}}{2}\left(\frac{y \sqrt{s}}{y^{2}+s}+\frac{\pi}{4}-I\left(\frac{y}{\sqrt{y^{2}+s}}\right)\right) .
\end{aligned}
$$

It can be checked that $I\left(y / \sqrt{y^{2}+s}\right) \geq y \sqrt{s} /\left(y^{2}+s\right)$, and hence

$$
\left|\tilde{W}_{s}\right| \leq \frac{\pi \sigma \sqrt{R_{s}^{2}+1}}{8} .
$$

This implies that $R_{s} \leq \pi \sigma \sqrt{R_{s}^{2}+1} / 8$ and hence that

$$
R_{s} \leq \frac{\pi \sigma}{\sqrt{64-\pi^{2} \sigma^{2}}}
$$

which proves the proposition.

Now part (ii) of Theorem 1.1 follows by (2.7).

Remark. At first it might seem that the statement $\left|W_{s}(i y)\right| \leq K$ for $y>0$ is stronger than $\mathcal{C}_{t, \sigma} \leq K$, but in fact, they are equivalent. Indeed, the curve $\left(f_{t}\left(\lambda_{t}+\right.\right.$ $i y), y>0)$ is the curve generated by the driving function $\lambda_{s}^{*}=\lambda_{s \wedge t}$, which is clearly a Lip- $\frac{1}{2}$ function. Thus, the condition $\mathcal{C}_{t, \sigma} \leq K$ implies that $\left|W_{s}(i y)\right| \leq K$.

\section{A bound in the range $0 \leq \sigma<4$}

This section is devoted to the proof of the following proposition, which will give part (i) of Theorem 1.1, as in the previous section.

Proposition 4.1. Let $\lambda \in \Lambda_{\sigma}$ with $\sigma<4$, fix some $t>0$ and define $\hat{h}_{s}(i y)=$ $X_{s}(i y)+i Y_{s}(i y)$ as the solution to (2.2). Then, for all $s \in[0, t]$ and $y>0$, we have

$$
\left|W_{s}(i y)\right|=\left|\frac{X_{s}(i y)}{Y_{s}(i y)}\right| \leq L_{\sigma} .
$$

The proof of Proposition 4.1 requires more work than that of Proposition 3.1. In proving this, we shall employ the time-change of Section 2.1 and bound $X_{s}$ and $\theta_{s}$ by comparing them to some properly chosen functions, using a version of the Grönwall inequality, which we now state and prove. 
Proposition 4.2. (Grönwall's inequlity) Let $F(t, x)$ be a bounded, continuous function, which is increasing and continuously differentiable in the variable $x$. Let $U$ be a continuous function on an interval $[0, T]$ such that

$$
U_{t} \leq \int_{0}^{t} F\left(r, U_{r}\right) d r
$$

for every $t \in[0, T]$. If $V$ is a continuous function defined on $[0, T]$, satisfying

$$
V_{t}=\int_{0}^{t} F\left(r, V_{r}\right) d r
$$

for $t \in[0, T]$, then $U_{t} \leq V_{t}$ for all $t \in[0, T]$.

Proof. We have that

$$
U_{t}-V_{t} \leq \int_{0}^{t}\left(F\left(r, U_{r}\right)-F\left(r, V_{r}\right)\right) d r=\int_{0}^{t} \Theta(r)\left(U_{r}-V_{r}\right) d r
$$

where, writing $F^{\prime}(t, x)=\partial_{x} F(t, x)$,

$$
\Theta(r)=\int_{0}^{1} F^{\prime}\left(r, p U_{r}+(1-p) V_{r}\right) d p
$$

By the continuity of $U, V$ and $F^{\prime}$, together with the dominated convergence theorem, we have that $\Theta$ is continuous. Moreover, $F$ is increasing, that is, $F^{\prime}$ is nonnegative and it thus follows that $\Theta$ is nonnegative. Hence, the claim follows from the standard Grönwall inequality, since $U_{0}-V_{0} \leq 0$.

Remark. We can actually relax the assumptions on $F$ somewhat and allow it to blow up at a point, say 0 . Then $V_{t}$ still dominates $U_{t}$, at least until the first time $U$ hits 0 . More precisely, the following holds by the very same proof. Let $t_{U}^{\delta}=\inf \left\{t \in[0, T]: U_{t}=\delta\right\}$ and $t_{V}^{\delta}=\inf \left\{t \in[0, T]: V_{t}=\delta\right\}$. Assume that for each $t, F(t, x)$ is increasing and continuously differentiable on $(0, \infty)$ in the variable $x$. In particular, $F^{\prime}(t, x)$ is bounded on every compact subset of $[0, \infty) \times(0, \infty)$. If $U_{t}$ and $V_{t}$ are continuous,

$$
U_{t} \leq U_{0}+\int_{0}^{t} F\left(r, U_{r}\right) d r
$$

for $t<t_{U}^{0}$,

$$
V_{t}=V_{0}+\int_{0}^{t} F\left(r, V_{r}\right) d r
$$

for $t<t_{V}^{0}$ and $U_{0} \leq V_{0}$, then for each $0<\delta<U_{0}$ it holds that for all $t<\min \left(t_{U}^{\delta}, t_{V}^{\delta}\right)$, $U_{t} \leq V_{t}$. Moreover, this implies that $t_{U}^{\delta} \leq t_{V}^{\delta}$.

We now discuss the bound on $X_{s}$. Consider (2.8) and note that $W_{s}^{2} /\left(W_{s}^{2}+1\right) \leq 1$ for all $s$ and that $\beta \in \Lambda_{\sigma}$. Hence, it makes sense to compare $X_{s}$ with solutions to the equation

$$
d Z_{s}=\sigma d \sqrt{s}-\frac{c}{Z_{s}} d s, \quad Z_{0}=z_{0}
$$

We will carry out this analysis for functions $X_{s}$ that have strayed from 0 , and hence, by symmetry, it will only be necessary for us to consider solutions to (4.2) started from $z_{0}>0$. The solution of (4.2) depends heavily on the value of $c$ and we shall only need the solution for $c=\sigma^{2} / 8$, as will be seen later. 
Lemma 4.3. The solution, $Z_{s}$, to (4.2) with $c=\sigma^{2} / 8$ and $z_{0}>0$ exists for all $s \geq 0$ and is given by

$$
Z_{s}=\frac{\sigma \sqrt{s}}{2}+z_{0} \exp \left\{\mathcal{W}\left(\frac{\sigma \sqrt{s}}{2 z_{0}}\right)\right\}
$$

where $\mathcal{W}$ is the Lambert $\mathcal{W}$ function, that is, the nonnegative solution to the equation

$$
\mathcal{W}(x) e^{\mathcal{W}(x)}=x .
$$

Proof. First, we note that $\mathcal{W}(0)=0$ implies that $Z_{0}=z_{0}$. Next, using that

$$
\frac{d \mathcal{W}}{d x}(x)=\frac{1}{1+\mathcal{W}(x)} e^{-\mathcal{W}(x)},
$$

we have

$$
\begin{aligned}
\frac{d Z_{s}}{d s} & =\frac{\sigma}{4 \sqrt{s}}+\frac{1}{1+\mathcal{W}\left(\frac{\sigma \sqrt{s}}{2 z_{0}}\right)} \frac{\sigma}{4 \sqrt{s}}=\frac{\sigma}{2 \sqrt{s}}-\frac{\sigma}{4 \sqrt{s}} \frac{\mathcal{W}\left(\frac{\sigma \sqrt{s}}{2 z_{0}}\right)}{1+\mathcal{W}\left(\frac{\sigma \sqrt{s}}{2 z_{0}}\right)} \\
& =\frac{\sigma}{2 \sqrt{s}}-\frac{\sigma}{4 \sqrt{s}} \frac{1}{1+\frac{2 z_{0}}{\sigma \sqrt{s}} \exp \left(\mathcal{W}\left(\frac{\sigma \sqrt{s}}{2 z_{0}}\right)\right)} \\
& =\frac{\sigma}{2 \sqrt{s}}-\frac{\sigma^{2}}{8} \frac{1}{\frac{\sigma \sqrt{s}}{2}+z_{0} \exp \left(\mathcal{W}\left(\frac{\sigma \sqrt{s}}{2 z_{0}}\right)\right)}=\frac{\sigma}{2 \sqrt{s}}-\frac{\sigma^{2}}{8} \frac{1}{Z_{s}}
\end{aligned}
$$

and thus we are done.

We now turn to the bound for $\theta_{s}$. For $x_{0}>0$ and $\sigma<4$ we define the following function

$$
H_{x_{0}}(x)=\left(\frac{\sigma \sqrt{x}}{2}+x_{0} \exp \left\{\mathcal{W}\left(\frac{\sigma \sqrt{x}}{2 x_{0}}\right)\right\}\right)^{2}=\frac{\sigma^{2} x}{4}\left(1+\frac{1}{\mathcal{W}\left(\frac{\sigma \sqrt{x}}{2 x_{0}}\right)}\right)^{2}
$$

For $\kappa \in\left(\frac{\sigma^{2}}{4}, 4\right)$, let $M=M(\kappa)$ be such that

$$
\frac{\sigma^{2}}{4}\left(1+\frac{1}{\mathcal{W}\left(\frac{\sigma \sqrt{M}}{2 x_{0}}\right)}\right)^{2}=\kappa
$$

Since $\mathcal{W}$ is an increasing function, it follows that $H_{x_{0}}(x) \leq \kappa x$ for $x \geq M$.

Lemma 4.4. Fix $x_{0}, y_{0}>0$ and $\sigma<4$ and let $V_{s}$ denote the solution to the differential equation

$$
\frac{d V}{d s}=\frac{1}{4} \frac{H_{x_{0}}\left(V_{s}\right)}{y_{0}^{2}+s}+\frac{1}{4}, \quad V_{0}=0 .
$$

Then, for any $\kappa \in\left(\frac{\sigma^{2}}{4}, 4\right)$,

$$
V_{s} \leq \max \left(\frac{M}{y_{0}^{2}}, \frac{1}{4-\kappa}\right)\left(y_{0}^{2}+s\right),
$$

where $M$ and $\kappa$ are related as in (4.5). 
Proof. By (4.6), $V$ is continuous and strictly increasing to $\infty$. Thus $\tau=\inf \{s>$ $\left.0: V_{s} \geq M\right\}$ is finite and for $s \leq \tau, V_{s} \leq M$. For $s>\tau$, we have that

$$
\frac{d V_{s}}{d s} \leq \frac{\kappa}{4} \frac{V_{s}}{y_{0}^{2}+s}+\frac{1}{4}
$$

since $H_{x_{0}}(x) \leq \kappa x$ for $x \geq M$ and thus

$$
V_{s}-V_{\tau} \leq \int_{\tau}^{s}\left(\frac{\kappa}{4} \frac{V_{r}}{y_{0}^{2}+r}+\frac{1}{4}\right) d r
$$

Let $N_{s}$ be the solution to the differential equation

$$
\frac{d N_{s}}{d s}=\frac{\kappa}{4} \frac{N_{s}}{y_{0}^{2}+s}+\frac{1}{4}, \quad s>\tau
$$

given $N_{\tau}$. Then for $s>\tau$,

$$
N_{s}=N_{\tau}\left(\frac{y_{0}^{2}+s}{y_{0}^{2}+\tau}\right)^{\frac{\kappa}{4}}+\frac{1}{4-\kappa}\left(y_{0}^{2}+s\right)-\frac{1}{4-\kappa}\left(y_{0}^{2}+s\right)^{\frac{\kappa}{4}}\left(y_{0}^{2}+\tau\right)^{1-\frac{\kappa}{4}},
$$

and by Proposition 4.2,

$$
V_{s} \leq V_{\tau}\left(\frac{y_{0}^{2}+s}{y_{0}^{2}+\tau}\right)^{\frac{\kappa}{4}}+\frac{1}{4-\kappa}\left(y_{0}^{2}+s\right)-\frac{1}{4-\kappa}\left(y_{0}^{2}+s\right)^{\frac{\kappa}{4}}\left(y_{0}^{2}+\tau\right)^{1-\frac{\kappa}{4}} .
$$

Thus,

$$
\frac{V_{s}}{y_{0}^{2}+s}-\frac{1}{4-\kappa} \leq\left(\frac{y_{0}^{2}+s}{y_{0}^{2}+\tau}\right)^{\frac{\kappa}{4}-1}\left(\frac{V_{\tau}}{y_{0}^{2}+\tau}-\frac{1}{4-\kappa}\right) .
$$

Since $y_{0}^{2}+\tau \leq y_{0}^{2}+s$, this implies that either

$$
\frac{V_{s}}{y_{0}^{2}+s}-\frac{1}{4-\kappa} \leq 0 \quad \text { or } \quad \frac{V_{s}}{y_{0}^{2}+s} \leq \frac{V_{\tau}}{y_{0}^{2}+\tau} \leq \frac{M}{y_{0}^{2}}
$$

Thus we have that for $s>\tau$,

$$
V_{s} \leq \max \left(\frac{M}{y_{0}^{2}}, \frac{1}{4-\kappa}\right)\left(y_{0}^{2}+s\right)
$$

which, together with the bound $V_{s} \leq M$ for $s \leq \tau$, concludes the proof.

We are now ready to prove Proposition 4.1.

Proof of Proposition 4.1. Fix $\sigma<4$ and $t>0$ and let $K_{\sigma}=\sigma / \sqrt{16-\sigma^{2}}$. If $\left|W_{s}\right| \leq K_{\sigma}$ for all $s \leq t$, we are done, since $K_{\sigma} \leq L_{\sigma}$. Assume the contrary and let $s_{1}>0$ be such that $\left|W_{s_{1}}\right|>K_{\sigma}$. By symmetry, we may assume that $W_{s_{1}}>0$. Let $s_{0}=\sup \left\{s<s_{1}: W_{s} \leq K_{\sigma}\right\}$ and write $\left(\widehat{X}_{s}, \widehat{Y}_{s}\right):=\left(X_{s_{0}+s}, Y_{s_{0}+s}\right)$ for $s \in\left[0, s_{1}-s_{0}\right]$. Then, by (2.8),

$$
\widehat{X}_{s}=\widehat{X}_{0}+\beta_{s_{0}+s}-\beta_{s_{0}}-\int_{0}^{s} \frac{2 W_{s_{0}+r}^{2}}{W_{s_{0}+r}^{2}+1} \frac{1}{\widehat{X}_{r}} d r \leq \widehat{X}_{0}+\sigma \sqrt{s}-\frac{\sigma^{2}}{8} \int_{0}^{s} \frac{1}{\widehat{X}_{r}} d r,
$$

since $w \mapsto w^{2} /\left(w^{2}+1\right)$ is an increasing function and $W_{s_{0}+r} \geq K_{\sigma}$ for $r \in\left[0, s_{1}-s_{0}\right]$. By the remark after Proposition 4.2, together with Lemma 4.3,

$$
\widehat{X}_{s} \leq \frac{\sigma \sqrt{s}}{2}+\widehat{X}_{0} \exp \left\{\mathcal{W}\left(\frac{\sigma \sqrt{s}}{2 \widehat{X}_{0}}\right)\right\}
$$


Next, we note that

$$
d \widehat{Y}_{s}^{2}=\frac{4 \widehat{Y}_{s}^{2}}{\widehat{X}_{s}^{2}+\widehat{Y}_{s}^{2}} d s, \quad \widehat{Y}_{0}=Y_{s_{0}} .
$$

Reparametrizing as in Section 2.1, that is, defining $\theta_{s}$ as the inverse function of $\widehat{Y}_{s}^{2}-Y_{s_{0}}^{2}$, we have

$$
\theta_{s}=\frac{1}{4} \int_{0}^{s}\left(\frac{\widehat{X}_{\theta_{r}}^{2}}{Y_{s_{0}}^{2}+r}+1\right) d r \leq \frac{1}{4} \int_{0}^{s}\left(\frac{H_{X_{s_{0}}}\left(\theta_{r}\right)}{Y_{s_{0}}^{2}+r}+1\right) d r
$$

where $H_{X_{s_{0}}}$ is defined as in (4.4). Thus, by Proposition 4.2, $\theta_{s} \leq V_{s}$ where $V_{s}$ is the solution to (4.6) with $\left(x_{0}, y_{0}\right)=\left(X_{s_{0}}, Y_{s_{0}}\right)$. By Lemma 4.4, we have

$$
\theta_{s} \leq \max \left(\frac{M}{Y_{s_{0}}^{2}}, \frac{1}{4-\kappa}\right)\left(Y_{s_{0}}^{2}+s\right)
$$

where $\kappa \in\left(\frac{\sigma^{2}}{4}, 4\right)$ and $M=M(\kappa)$ is defined by (4.5). Moreover,

$$
\frac{\widehat{X}_{\theta_{s}}^{2}}{\widehat{Y}_{\theta_{s}}^{2}}=\frac{\widehat{X}_{\theta_{s}}^{2}}{Y_{s_{0}}^{2}+s} \leq \frac{H_{X_{s_{0}}}\left(\theta_{s}\right)}{Y_{s_{0}}^{2}+s} \text {. }
$$

If $\theta_{s} \leq M$, then

$$
\frac{H_{X_{s_{0}}}\left(\theta_{s}\right)}{Y_{s_{0}}^{2}+s} \leq \frac{H_{X_{s_{0}}}(M)}{Y_{s_{0}}^{2}+s}=\frac{\kappa M}{Y_{s_{0}}^{2}+s} \leq \frac{\kappa M}{Y_{s_{0}}^{2}} .
$$

Recalling that $H_{X_{s_{0}}}(x) \leq \kappa x$ for $x \geq M$, we have that if $\theta_{s}>M$, then

$$
\frac{H_{X_{s_{0}}}\left(\theta_{s}\right)}{Y_{s_{0}}^{2}+s} \leq \frac{\kappa \theta_{s}}{Y_{s_{0}}^{2}+s} \leq \max \left(\frac{\kappa M}{Y_{s_{0}}^{2}}, \frac{\kappa}{4-\kappa}\right) \text {. }
$$

Thus,

$$
\frac{\left|X_{s_{1}}\right|}{Y_{s_{1}}} \leq \max \left(\frac{\sqrt{\kappa} \sqrt{M}}{Y_{s_{0}}}, \frac{\sqrt{\kappa}}{\sqrt{4-\kappa}}\right) .
$$

Finally, we minimize (4.9), as a function of $\kappa$. Note that this is achieved for $\kappa$ such that

$$
\frac{\kappa M(\kappa)}{Y_{s_{0}}^{2}}=\frac{\kappa}{4-\kappa} .
$$

Since $X_{s_{0}} / Y_{s_{0}}=K_{\sigma}$, we obtain $L_{\sigma}$ as defined by (1.4), which concludes the proof.

\section{Regularity and winding rates}

5.1. Hölder exponents. This section proves Corollaries 1.2 and 1.3, that is, we estimate the Hölder exponents for the curve $\gamma \in \Gamma_{\sigma}$ depending on $\sigma$. The arguments given here are standard, but we choose to give short derivations here for the convenience of the reader. In this section, constants may vary between the lines, even though they are denoted in the same way.

Proof of Corollary 1.2 and Corollary 1.3. Fix $\sigma<4,0<y \leq 1, \varepsilon>0$ and $\varepsilon \leq t \leq 1$ and let $s \in\left[0, y^{2}\right]$. First, we note that

$$
\begin{aligned}
|\gamma(t+s)-\gamma(t)| \leq & \left.\mid \gamma(t+s)-f_{t+s}\left(\lambda_{t+s}+i y\right)\right)|+| \gamma(t)-f_{t}\left(\lambda_{t}+i y\right) \mid \\
& +\left|f_{t+s}\left(\lambda_{t+s}+i y\right)-f_{t+s}\left(\lambda_{t}+i y\right)\right|+\left|f_{t+s}\left(\lambda_{t}+i y\right)-f_{t}\left(\lambda_{t}+i y\right)\right| .
\end{aligned}
$$


The first two terms we bound as follows. Since $\gamma(t)=f_{t}\left(\lambda_{t}+i 0^{+}\right)$, we have

$$
\left|\gamma(t)-f_{t}\left(\lambda_{t}+i y\right)\right| \leq \int_{0}^{y}\left|f_{t}^{\prime}\left(\lambda_{t}+i r\right)\right| d r
$$

Moreover, we let

$$
m_{\sigma}= \begin{cases}\min \left(L_{\sigma}, \frac{\pi \sigma}{\sqrt{64-\pi^{2} \sigma^{2}}}\right), & \text { if } \sigma<\frac{8}{\pi} \\ L_{\sigma} & \text { if } \sigma \geq \frac{8}{\pi}\end{cases}
$$

and write $\xi_{\sigma}=\left(m_{\sigma}^{2}-1\right) /\left(m_{\sigma}^{2}+1\right) \in(-1,1)$. By $(2.9)$ and the fact that the function $x \mapsto\left(x^{2}-1\right) /\left(x^{2}+1\right)$ is increasing on $(0, \infty)$ together with Proposition 3.1 and Proposition 4.1 we have that

$$
\left|f_{t}^{\prime}\left(\lambda_{t}+i r\right)\right|=\exp \left\{\int_{0}^{t} \frac{W_{u}^{2}-1}{W_{u}^{2}+1} d \log Y_{u}\right\} \leq Y_{t}^{\xi_{\sigma}} r^{-\xi_{\sigma}} .
$$

By (2.5), we have that for $u \in[0, s]$,

$$
Y_{t+u}^{\xi_{\sigma}} \leq C(\sigma, \varepsilon)
$$

since $s, t \leq 1$ and therefore

$$
\int_{0}^{y}\left|f_{t}^{\prime}\left(\lambda_{t}+i r\right)\right| d r \leq C(\sigma, \varepsilon) \int_{0}^{y} r^{-\xi_{\sigma}} d r=\frac{C(\sigma, \varepsilon)}{1-\xi_{\sigma}} y^{1-\xi_{\sigma}} .
$$

The same statement holds with $t+s$ in place of $t$ and thus we have a bound on the first two terms of (5.1). Let $I_{s, t}^{y}$ denote the line segment connecting $\lambda_{t}+i y$ to $\lambda_{t+s}+i y$. Using the distortion theorem, (5.3) and that $\sqrt{s} \leq y$, we have that

$$
\begin{aligned}
\left|f_{t+s}\left(\lambda_{t+s}+i y\right)-f_{t+s}\left(\lambda_{t}+i y\right)\right| & \leq\left|\lambda_{t+s}-\lambda_{t}\right| \max _{w \in I_{s, t}^{y}}\left|f_{t+s}^{\prime}(w)\right| \leq \sigma \sqrt{s} \max _{w \in I_{s, t}^{y}}\left|f_{t+s}^{\prime}(w)\right| \\
& \leq C(\sigma) y\left|f_{t+s}^{\prime}\left(\lambda_{t+s}+i y\right)\right| \leq C y^{1-\xi_{\sigma}} .
\end{aligned}
$$

Finally, since $s \in\left[0, y^{2}\right]$, Lemma 3.5 of [6] and (5.3) imply that

$$
\left|f_{t+s}\left(\lambda_{t}+i y\right)-f_{t}\left(\lambda_{t}+i y\right)\right| \leq C y\left|f_{t}^{\prime}\left(\lambda_{t}+i y\right)\right| \leq C y^{1-\xi_{\sigma}} .
$$

Thus, by (5.1) and the above inequalities, letting $s=y^{2}$, we have

$$
|\gamma(t+s)-\gamma(t)| \leq C(\sigma, \varepsilon) s^{\frac{1}{1+m_{\sigma}^{2}}}
$$

that is, $\gamma$ is Hölder continuous with exponent $\alpha$ for each

$$
\alpha \leq \frac{1}{1+m_{\sigma}^{2}}
$$

and this concludes the proof.

Remark. We have seen that the Hölder exponent is determined by the behavior of the derivative near the tip of the curve, which in turn is estimated using (2.9). The derivative can be explicitly bounded if the reverse flow stays in a particular cone so that $\sup \left|W_{r}\right| \leq C$. However, the sharp behavior depends on the integrated values of $\left(W_{r}^{2}-1\right) /\left(W_{r}^{2}+1\right)$ and we expect that the optimal bound on the integral is strictly smaller than the integrated optimal $L^{\infty}$-bound. In the case of SLE, there is of course no almost sure $L^{\infty}$-bound, but the process $W_{r}$ has an invariant distribution in an appropriately weighted measure, and (2.9) can be precisely estimated using this invariant distribution and an intermediate deviations argument, see [6]. In the case of a general Lip- $\frac{1}{2}$ function these techniques are not available. 
5.2. Winding rates. Theorem 1.1 also easily implies estimates on the winding rate at the tip of the curve $\gamma \in \Gamma_{\sigma}$ depending on $\sigma$, that is, we obtain estimates on the growth rate of $\left|\arg f_{t}^{\prime}\left(\lambda_{t}+i y\right)\right|$ as $y$ tends to 0 . Geometrically, this measures the winding of the hyperbolic geodesic from $\gamma(t)$ to $\infty$ in $\mathbf{H} \backslash \gamma([0, t])$, close to the tip $\gamma(t)$ when the geodesic is parametrized by harmonic measure. By (2.10) and (2.5) we have a trivial bound

$$
\left|\arg f_{t}^{\prime}\left(\lambda_{t}+i y\right)\right| \leq 2 \log \frac{Y_{t}}{y} \leq \log \left(y^{2}+4 t\right)+2 \log \left(y^{-1}\right),
$$

since $2\left|W_{s}\right| /\left(W_{s}^{2}+1\right) \leq 1$. By the easy estimate $\mathcal{C}_{t, \sigma} \leq \sigma / \sqrt{4-\sigma^{2}}$ of [13], we get a non-trivial bound in the case $\sigma<\sqrt{2}$. By virtue of Corollary 1.3, we have the following improvement for $\sigma<4 \sqrt{2} / \pi$.

Proposition 5.1. Let $\gamma \in \Gamma_{\sigma}$ with $\sigma<4 \sqrt{2} / \pi$. Then

$$
\left|\arg f_{t}^{\prime}\left(\lambda_{t}+i y\right)\right| \leq \frac{\pi \sigma \sqrt{64-\pi^{2} \sigma^{2}}}{64} \log \left(y^{2}+4 t\right)+\frac{\pi \sigma \sqrt{64-\pi^{2} \sigma^{2}}}{32} \log y^{-1}
$$

Proof. By (3.1) and since $\sigma<4 \sqrt{2} / \pi$, we have that

$$
\left|W_{s}(i y)\right| \leq \frac{\pi \sigma}{\sqrt{64-\pi^{2} \sigma^{2}}}<1 .
$$

By (2.10), Proposition 3.1, Proposition 4.1, (2.5) and that the function $x \mapsto x /\left(x^{2}+1\right)$ is increasing on $[0,1]$, we have that

$$
\begin{aligned}
\left|\arg f_{t}^{\prime}\left(\lambda_{t}+i y\right)\right| & \leq 2 \int_{0}^{t} \frac{\left|W_{r}\right|}{W_{r}^{2}+1} d \log Y_{r} \leq 2 \frac{\pi \sigma \sqrt{64-\pi^{2} \sigma^{2}}}{64} \log \left(\frac{Y_{t}}{y}\right) \\
& \leq \frac{\pi \sigma \sqrt{64-\pi^{2} \sigma^{2}}}{64} \log \left(y^{2}+4 t\right)+\frac{\pi \sigma \sqrt{64-\pi^{2} \sigma^{2}}}{32} \log \left(y^{-1}\right)
\end{aligned}
$$

which is the desired estimate.

\section{Additional remarks}

In this section we collect a few simple observations that follow essentially directly from known results. For a curve $\gamma$, let us consider the optimal Hölder exponent achievable through reparametrization:

$$
\hat{\alpha}(\gamma)=\sup \{\alpha: \gamma \text { can be reparametrized to be Hölder- } \alpha\} \text {. }
$$

By Corollary 1.3, for small $\sigma$, we know that

$$
\hat{\alpha}\left(\Gamma_{\sigma}\right):=\inf \left\{\hat{\alpha}(\gamma): \gamma \in \Gamma_{\sigma}\right\} \geq 1-\pi^{2} \sigma^{2} / 64
$$

which, as remarked, immediately gives an upper bound on the maximal dimension for $\gamma \in \Gamma_{\sigma}$. However, under weak regularity assumptions, satisfied by $\gamma \in \Gamma_{\sigma}$, the optimal estimate is equal to the maximal reciprocal Minkowski dimension. Recall that a $k$ quasiarc is the image of a line segment under a $k$-quasiconformal homeomorphism of C.

Proposition 6.1. Suppose $\gamma$ is a quasiarc with Minkowski dimension $d_{M}$. Then

$$
\hat{\alpha}(\gamma)=d_{M}^{-1}
$$

In particular, if $\gamma$ is a $k$-quasiarc, then

$$
\hat{\alpha}(\gamma) \geq \frac{1}{1+k^{2}}
$$


Remark. The second statement uses Smirnov's result [14], but in fact, for small $k$, Ivrii's recent stronger result [5] stating that the dimension of a $k$-quasiarc is at most $1+\Sigma^{2} k^{2}+O\left(k^{8 / 3-\varepsilon}\right)$ for small $k$, combined with Hedenmalm's estimate $\Sigma<1$ [4] gives a better bound for small $k$.

Sketch of proof. The first statement follows from the ideas of Section 2 of [1]. The second assertion follows from the first, combined with Smirnov's result on the maximal Hausdorff dimension of a $k$-quasiarc, and the fact, due to Astala (see Theorem 1.5 of [2]), that the maximal Hausdorff dimension over the set of $k$ quasiarcs equals the corresponding maximal Minkowski dimension. (This is not true in general for a fixed quasiarc.) Let us sketch the proof of (6.1) following [1]. For a curve $\gamma$ and $\delta>0$, let $M(\gamma, \delta)$ be the minimal number of curve segments of diameter at most $\delta$ needed to cover $\gamma$. We first claim that if $M(\gamma, \delta) \lesssim \delta^{-r}$ then for any $r^{\prime}>r$ there is a Hölder- $1 / r^{\prime}$ parametrization of $\gamma$. Indeed, choosing first any parametrization, $\gamma(s), s \in[0,1]$, not constant on any subinterval, we may define the following reparametrization

$$
\tau(s)=\frac{\sum_{n} n^{-2} M\left(\gamma[0, s], 2^{-n}\right) 2^{-r n}}{\sum_{n} n^{-2} M\left(\gamma, 2^{-n}\right) 2^{-r n}} .
$$

Note that the denominator is bounded. Suppose $\varepsilon>0$ and $\left|\gamma\left(s_{1}\right)-\gamma\left(s_{2}\right)\right| \geq \varepsilon$. If $2^{-n}<\varepsilon$ and sufficiently small, then we have $M\left(\gamma\left[0, s_{2}\right], 2^{-n}\right)-M\left(\gamma\left[0, s_{1}\right], 2^{-n}\right) \geq 1$. Hence for all $\varepsilon>0$ small enough,

$$
\tau\left(s_{2}\right)-\tau\left(s_{1}\right) \geq C \sum_{n>-\log \varepsilon} n^{-2} 2^{-r n} \geq C^{\prime} \varepsilon^{r^{\prime}}
$$

On the other hand, we can estimate $M$ in terms of $d_{M}$ as follows: By the quasiarc property there is a constant $C_{\gamma}<\infty$ such that the following holds. Given $\delta>0$, partition $\gamma$ into segments of diameter $C_{\gamma} \delta$ by considering stopping times defined as follows: $t_{0}=0$ and then for $j=1,2, \ldots, N_{\delta}, t_{j}=\inf \left\{t \geq t_{j-1}:\left|\gamma(t)-\gamma\left(t_{j-1}\right)\right| \geq C_{\gamma} \delta\right\}$ (terminating if the end point of the curve is within distance $C_{\gamma} \delta$ ). Then given any cover of $\gamma$ by balls of diameter $\delta$, by the quasicircle property, any ball in this cover contains at most one of the partitioning points, $\left\{\gamma\left(t_{j}\right)\right\}$. Hence for any $r>d_{M}$, we obtain $M(\gamma, \delta) \leq N_{\delta} \lesssim \delta^{-r}$.

Given Proposition 6.1, we would like to relate $k$ and $\sigma$ quantitatively. Marshall and Rohde [11] show that these parameters are quantitatively related in the sense that $k \rightarrow 0$ as $\sigma \rightarrow 0$. In the other direction, taking $\lambda(t)=\sigma \sqrt{t}$ shows that one can not say anything for general quasiarcs, but if the dilatation of the quasiconformal homeomorphism of $\mathbf{H}$ defining a quasislit tends to 1 (so that $k \rightarrow 0$ ), it does follow that $\sigma \rightarrow 0$. However, beyond these observations, no estimates appear to be available.

As was pointed out to us by Rohde, a recent result of Tran can be used to get an estimate for small $\sigma$. Let us sketch the argument. Fix $\lambda \in \Lambda_{\sigma}$ with $\sigma<1 / 3$. Set $\tilde{\lambda}=\lambda /\left(3\|\lambda\|_{1 / 2}\right)$ so that $\|\tilde{\lambda}\|_{1 / 2}=1 / 3$. On the other hand, since $\tilde{\lambda} \in \Lambda_{1 / 3}$ we can use Theorem 1.3 of [15] to embed $\gamma^{\lambda}$ in a holomorphic motion compatible with the Loewner equation in the following sense. For each $\tau \in \mathbf{D}$, it is possible to solve the Loewner equation (1.1) with the complex driving term, $t \mapsto \tau \tilde{\lambda}_{t}$. The solution extends to a conformal map $g_{t}: \hat{\mathbf{C}} \backslash L_{t} \rightarrow \hat{\mathbf{C}} \backslash R_{t}$, where $L_{t}, R_{t}$ are both simple curves. The curve $L_{t}=L_{t}^{\tau}$ moves holomorphically with $\tau$, and so if we write $S=[0,2 i]$, then there exists a holomorphic motion of $S, f: \mathbf{D} \times S \rightarrow \mathbf{C}$, such that $f\left(3\|\lambda\|_{1 / 2}, S\right)=\gamma^{\lambda}$ as a set. By the generalized $\lambda$-lemma, $f$ extends to a holomorphic 
motion of $\mathbf{C}, F: \mathbf{D} \times \mathbf{C} \rightarrow \mathbf{C}$, and for each $\tau \in \mathbf{D}, F(\tau, \cdot): \mathbf{C} \rightarrow \mathbf{C}$ is a quasiconformal homeomorphism of dilatation at most $(1+|\tau|) /(1-|\tau|)$. Setting $\tau=3\|\lambda\|_{1 / 2}$ we conclude the following.

Proposition 6.2. Suppose $\gamma \in \Gamma_{\sigma}$ with $\sigma<1 / 3$. Then $\gamma$ is a $3 \sigma$-quasiarc.

\section{References}

[1] Aizenman, M., and A. Burchard: Hölder regularity and dimension bounds for random curves. - Duke Math. J. 99:3, 1999, 419-453.

[2] Astala, K.: Calderon's problem for Lipschitz classes and the dimension of quasicircles. - Rev. Mat. Iberoam. 4:3, 1988, 469-486.

[3] Gehring, F.: Characteristic properties of quasidisks. - Séminaire de mathématiques supérieures, Presses de l'Université de Montréal, 1982.

[4] Hedenmalm, H.: Bloch functions, asymptotic variance and geometric zero packing. arXiv:1602.03358, 2016.

[5] IVRII, O.: Quasicircles of dimension 1+ $k^{2}$ do not exist. - arXiv:1511.07240, 2015.

[6] Johansson Viklund, F., and G. F. Lawler: Optimal Hölder exponent for the SLE path. Duke Math. J. 159:3, 2011, 351-383.

[7] Lawler, G. F.: Conformally invariant processes in the plane. - Math. Surveys Monogr. 114, Amer. Math. Soc., Providence, RI, 2005.

[8] Lind, J.: A sharp condition for the Loewner equation to generate slits. - Ann. Acad. Sci. Fenn. Math. 30, 2005, 143-158.

[9] Lind, J., D. E. Marshall, and S. Rohde: Collisions and spirals of Loewner traces. Duke Math. J. 154:3, 2010, 527-573.

[10] Lind, J., and S. RoHDE: Spacefilling curves and phases of the Loewner equation. - Indiana Univ. Math. J. 61:6, 2012, 2231-2249.

[11] Marshall, D. E., and S. Rohde: The Loewner differential equation and slit mappings. - J. Amer. Math. Soc. 18:4, 2005, 763-778.

[12] RingQvist, C. The Loewner equation: an introduction and the winding of its trace. - KTH Mathematics Master's thesis, 2015.

[13] Rohde, S., H. Tran, and M. Zinsmeister: The Loewner equation and Lipschitz graphs. Rev. Mat. Iberoam. 34, 2018, 937-948.

[14] Smirnov, S.: Dimension of quasicircles. - Acta Math. 205, 2010, 189-197.

[15] Tran, H.: Loewner equation driven by complex-valued functions. - arXiv:1707.01023, 2017.

[16] WANG, Y. Equivalent descriptions of the loewner energy. - Invent. Math. 218, 2019, 573?-621.

Received 7 January $2020 \bullet$ Accepted 13 May 2020 